\title{
Application of Informatics for the Enhanced Management of Robotic Operations
}

\author{
Jungwan Hong, Yongjin (James) Kwon \\ Department of Industrial Engineering, Ajou University, Suwon, South Korea \\ Email:yk73@ajou.ac.kr
}

Received October 2015

\begin{abstract}
In the past, the proposed concept-the application of informatics for robot operations-was difficult to implement due to technological limitations. With the advancement of ICT technologies, the production robots can be integrated within the information network, and the critical data can be processed to reveal the very important information. From the information, better managerial decisions are expected that will ultimately contribute to the organizations' efficiency and competitiveness. In this context, this study explains the merits of quality informatics in the domain of networked robots that are the essential tools for the global production companies. Even though this study merely presents the outcome of robot joint replacement period estimation, the foreseeable advantages of using the proposed method can be numerous. The future environment of global business will even push the boundaries of multinational, distributed production systems, and accelerate the implementation at a faster rate. Accordingly, the brand value of the product is expected to be greater than the country of the manufacturing. The survival of the company will be dependent on the optimized quality level that is encompassing the entire distributed production systems located throughout the whole. Under such circumstances, it can be foreseeable that the innovative ideas and the smart application of advanced technologies should be the priority.
\end{abstract}

\section{Keywords}

Informatics for Robotics, Process Information, Visibility, Data Processing, Robot Management

\section{Introduction}

In South Korea, smartphone and automobile companies are global operations, where a number of production facilities situated in foreign countries are involved. To increase their competitiveness, advanced automated production equipment, such as robots, became a critical component. The foreign production facilities are also interconnected with their headquarters, and the key production activities, such as defect rates and condition of production equipment, are being monitored 24 hours. One of the critical quality control activities is the robot maintenance. However, it is being resorted to the scheduled plan, and this contains the inherent risk of premature spending on replacement. Such is true even for today. Robot operators can hardly relate the robot conditions with the record of robot operations. This situation can be viewed as a consumer trying to purchase a used car without knowing any previous history. The lack of operational history data prohibits the users from taking the 
robot conditions into consideration [1]. In this context, the purpose of this study is to develop a novel quality informatics system for the networked production robots. The novelty includes 1) the application of informatics concept to the production robots in an effort to attain the component-level data that has not been previously considered; and 2) the robot usage data are directly linked to the managerial decision process that is defined as the quality informatics for the operational aspect of production robots. For that purpose, the proposed quality informatics system collects the robot status data from each axis motor, and process the data to extract the critical information that has not been possible in the past. The critical information is directly related to the decision of motor replacement period, which has a serious financial consequence to the organizations. The robot operational data are collected in real-time, from which the usage and status are monitored. The data represent the motor pulse signals, motor on/off time, motor malfunction data, and motor rotation data of each axis. The work cycle and idle time, and other ancillary data are also recorded in the database. Such data received from the component level have not been previously considered, when the product quality is of concern [2] [3]. Our initial analysis also shows that the robot programming can be adjusted to evenly assign the motion commands over all axes. Therefore, no premature or concentrated wear occurs on one axis, which can seriously hamper the robot positioning accuracy along specific directions. In the past, the inaccurate count for the movement of robot arm means that the robot status is not considered with the product quality. Now, with the ability to collect and analyze the robot axis motion data, a better managerial decision can be materialized. The underlying justification is that the product quality is affected by the equipment status. From the managerial aspects of robot operations, the engineers can instantly identify the robot status; hence a more pragmatic plan can be developed in terms of setting up a new production plan or initiating a critical maintenance for the robots. The overall idea is presented in Figure 1, depicting the new form of QC scheme that has evolved over the existing statistical techniques. This is centered on the notion of informatics [4] [5].

\section{The API Development}

To collect the data, the robot control system structure needs to be defined. It is shown in Figure 2. We developed the API (application programming interface) to extract the critical data, which in turn processed into important information. The information will be ultimately used in making a critical decision. The API connects remotely to the networked robots to perform the operations, such as programmed robot executions and robot servo motor on/off. The API extracts and analyzes the data from the robots, and displays the meaningful information to the operator. Finally, the API provides the real-time status as well as the replacement period of the robot axes. This provides only the critical information that directly contributes to the product quality. The structure of API is illustrated in Figure 3.
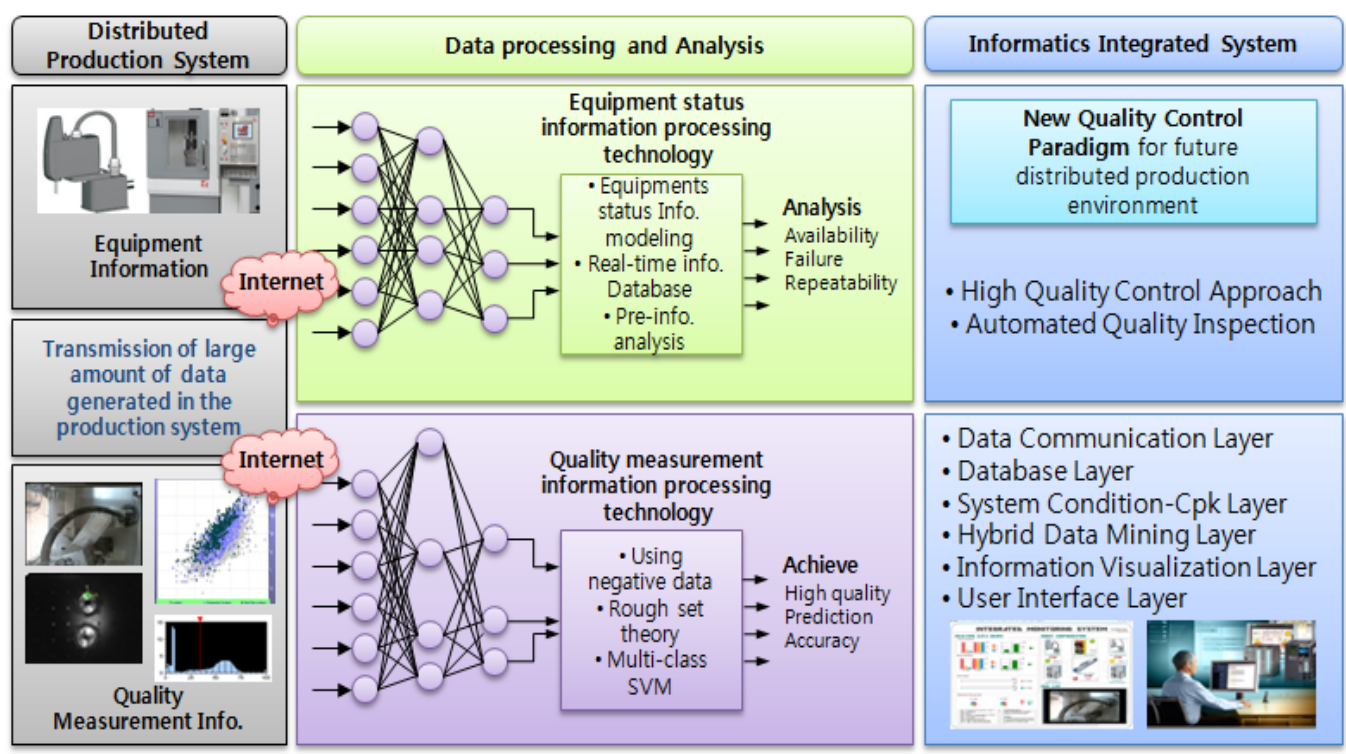

Figure 1. A concept of informatics-based quality control approach. 


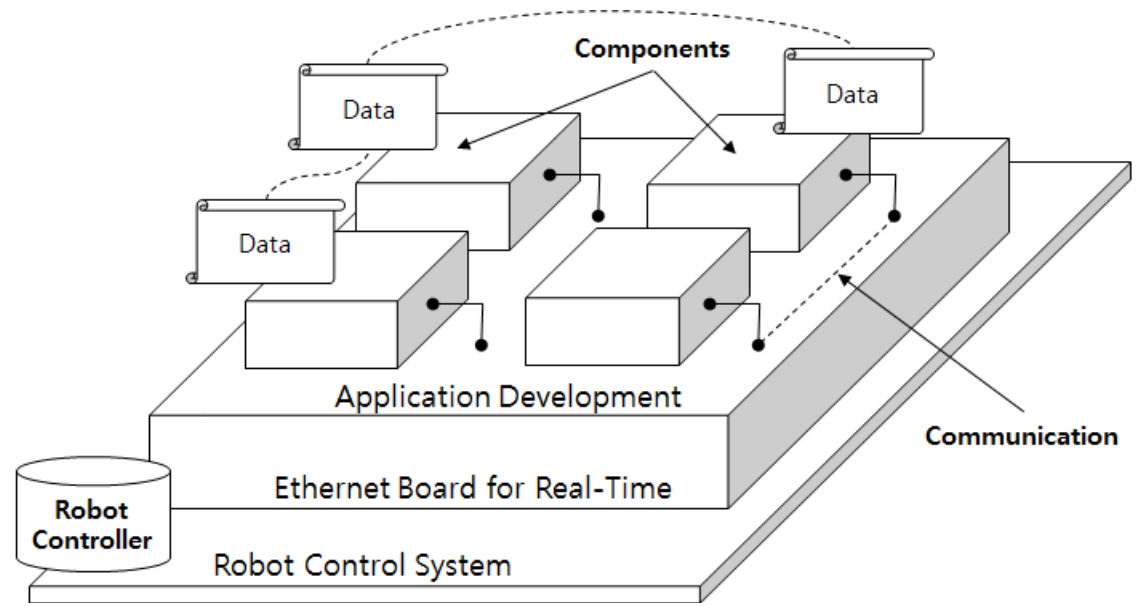

Figure 2. Robot control system structure.

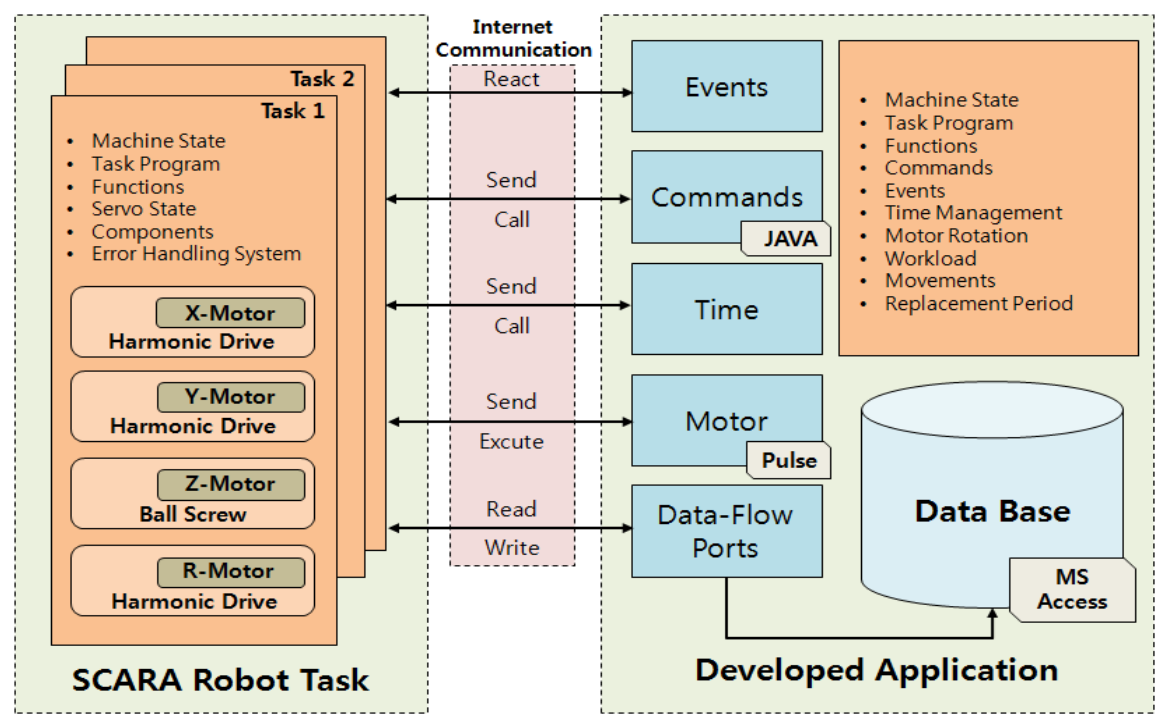

Figure 3. The workings of the application.

The API also executes a process of converting from the quality measurement information into knowledge. The quality measurement information to be handled in this layer can be classified in motor speed and various time data. The most notable quality information in this study is the number of revolutions of the motor/harmonic drive. To calculate the number of rotation of the motor/harmonic drive, the pulse movement values stored in the database for each-axis have to be trimmed. For the SCARA robot used in this study, the resolver pulse values are defined per one rotation of each axis. And speed reduction gear (harmonic drive) reduction ratio is also defined. Table 1 shows the reduction ratio and resolver pulse value per degrees of axis for the SCARA robot.

\section{Experimental Results}

To calculate the robot maintenance period, this study focuses on the most critical component, namely the harmonic drive assembly. The harmonic drive converts the torque from the electric servo motor into a higher value with the use of reduction gears, while at the same time, provides a structural rigidity between the motor and the rotating robot arm. Therefore, it should be replaced at a regular interval and the care must be exercised in order to precisely track the usage of it. The replacement period can be set at a regular interval.

For example, the user may replace it once every 6 year, or it can be replaced as a result of actual usage. It's much better to replace it based on actual usage, and in order to do that, the moving distance of each axis must be calculated. This process is shown in Figure 4. In the work envelope of $\mathrm{X} / \mathrm{Y}$ plane, when the robot moves from 


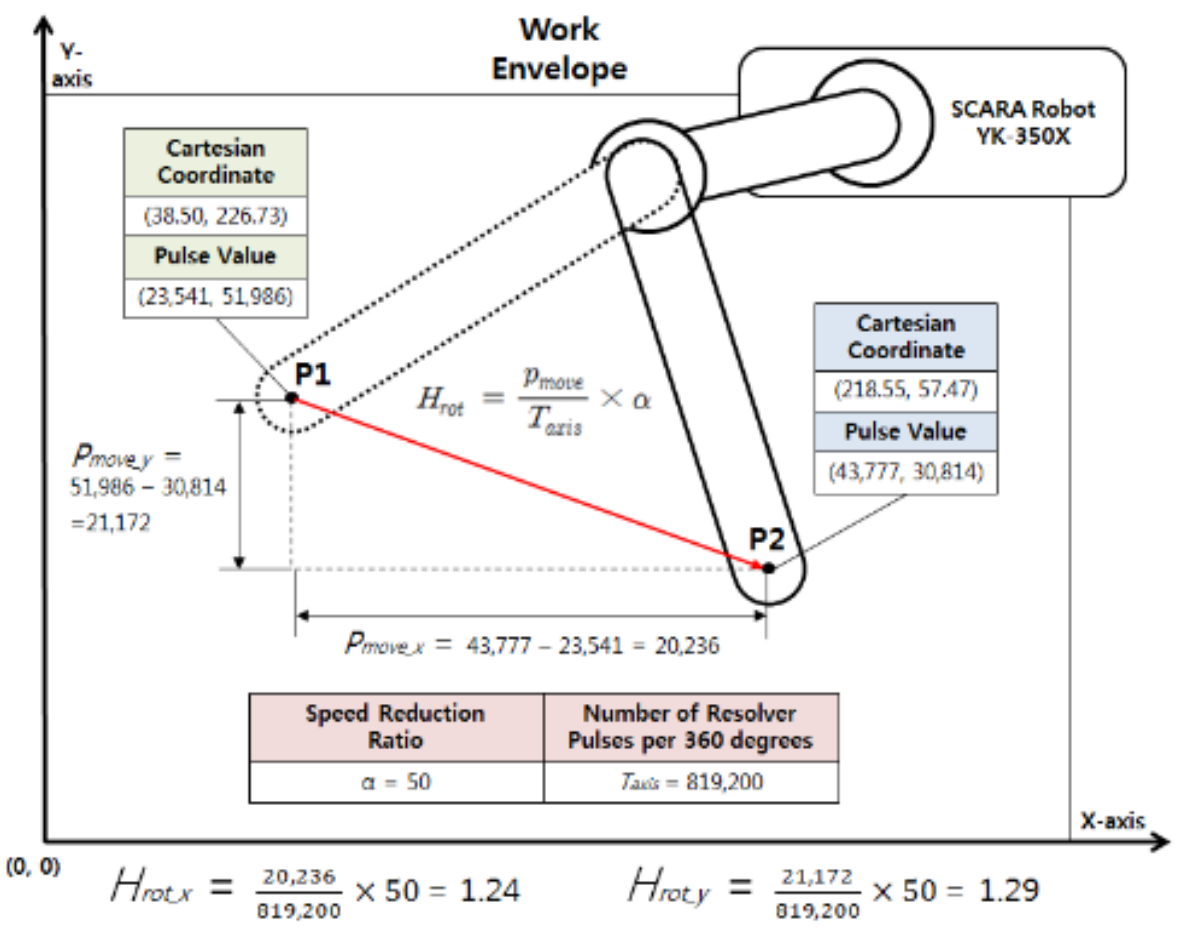

Figure 4. The workings of the application.

Table 1. Speed reduction ratio and resolver pulses per degrees/movement.

\begin{tabular}{cccc}
\hline Axis & Speed Reduction Ratio $(\alpha)$ & No. of Resolver Pulses per 360 Degrees & No. of Resolver Pulses per Lead Movement \\
\hline $\mathrm{X}$ & 50 & 819,200 & N/A \\
$\mathrm{Y}$ & 50 & 819,200 & N/A \\
$\mathrm{Z}$ & 50 & N/A & 16,384 \\
$\mathrm{R}$ & 50 & 819,200 & N/A \\
\hline
\end{tabular}

P1 to P2, the pulse variation values of $\mathrm{X} / \mathrm{Y}$-axis represent $P_{\text {move_x }}=20,236$ and $P_{\text {move_y }}=21,172$, respectively. B by applying the reduction ratio of 50 and the number of resolver pulses for 360 degrees (i.e., $T_{a x i s}=819,200$ ), it is possible to obtain the number of harmonic drive rotations. Then, the analytical results are shown to the operator. Figure 5 illustrates the algorithm and source code for the calculation. As shown, it processes the rotation and time data of motor/harmonic drive stored in the database. Then, the results are visually presented to the operator with analysis. Figure 6 illustrates some of the screen shots of the API. Integrated monitoring system indicates the current status of all components in the robot cell (two SCARA robots, one linear robot, a vision sensor, a web cam, etc.), while the second image shows the detailed usage information of the YK-220X SCARA robot. Just by simply looking at the display, it is possible to grasp the details of each robot at a component level. Figure 7(a) shows the results of motor rotation, using the original program instructions. Upon analysis, the operator can notice the excessive burden on the Y-axis motor, which will eventually wear out the Y-axis motor earlier than other motors. This also creates the positioning accuracy problem, when a specific motor accumulates too much rotational work.

To counter this problem, a minor adjustment to the program has been made to balance the work among the motors. With the new program, the robot has been running for many hours and the outcome is derived from the data. The result is illustrated in Figure 7(b), which shows the more even distribution of work among the axes in order to prevent an excessive wear on a single axis. At the same time, the replacement period has been substantially shortened, which will save a lot of money for the companies. The comparison is also provided in Table 2 and Table 3. 
TIME (unit Min)

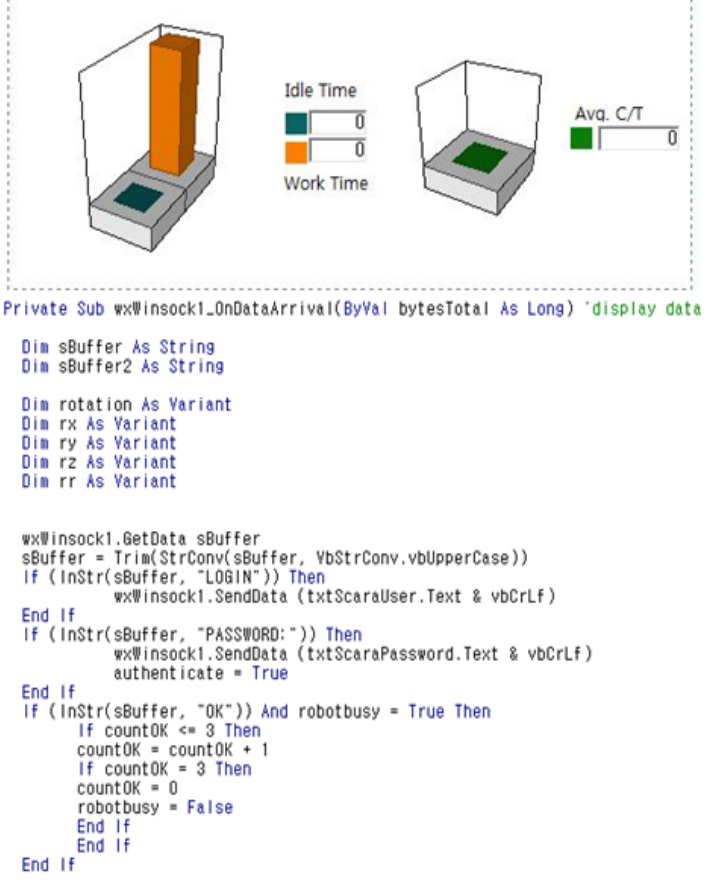

Figure 5. The source code and algorithm for calculation.

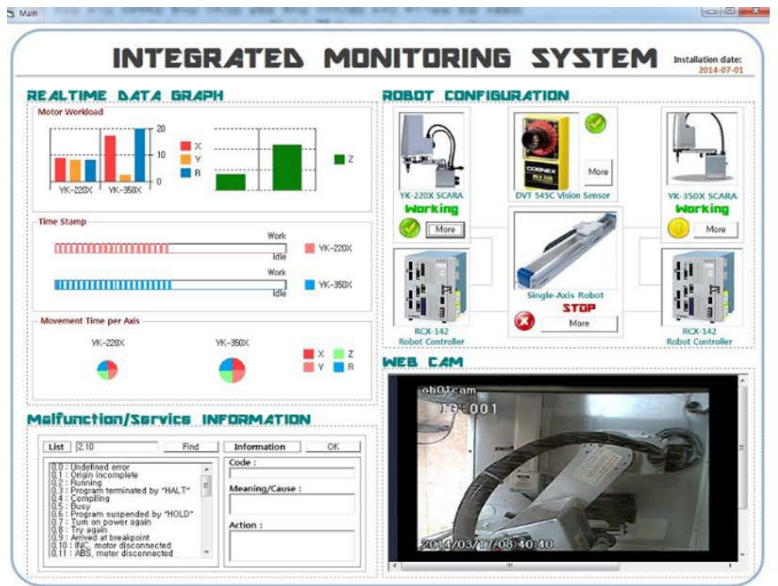

(a)

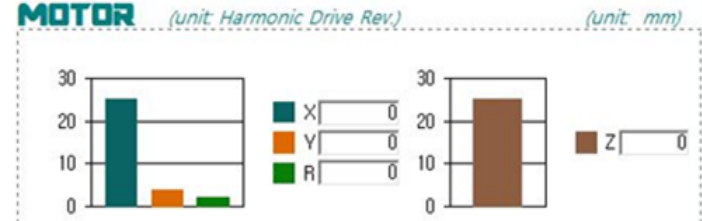

Private Sub cundscaraconnect_click() 'connection to Ethernet card

Dim nPort As Long

nPort $=$ txtscaraport. Text

If comdscaraconnect, Caption " "Connect" Then

sserver = Trin(txtscaralp, Text)

If Instr(sserver, $\left.": / / /^{*}\right)>0$ Then sserver $=$ Mid(sserver, Instr(sserver, $\left.": / / /^{*}\right)+3$ )

If $\operatorname{Instr}\left(\right.$ sserver, $\left.\psi^{*}\right)>1$ Then

spage = Mid (sserver, Instr (sserver, $" / *)$ )

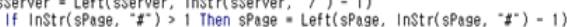

Else
spage.$\%$
End If

If Instr(sserver, ":" ) > 1 Then

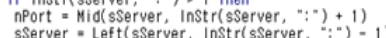
sserver
End If

wxlinsock1. Connect sServer, nPort 'connection to Ethernet card (sServer is IP address,

Else
vxllinsock1. SendData "LOGOUT" \& voCrLf

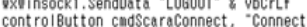

txtscarasend. Enabled $=$ False

cindscaraSend. Enabled $=\mathrm{False}$

candservoon. Enabled = False

extscaraport. Enabled = True

extscaraport. Enabled " Irve

txtscarapassvord. Enabled = True

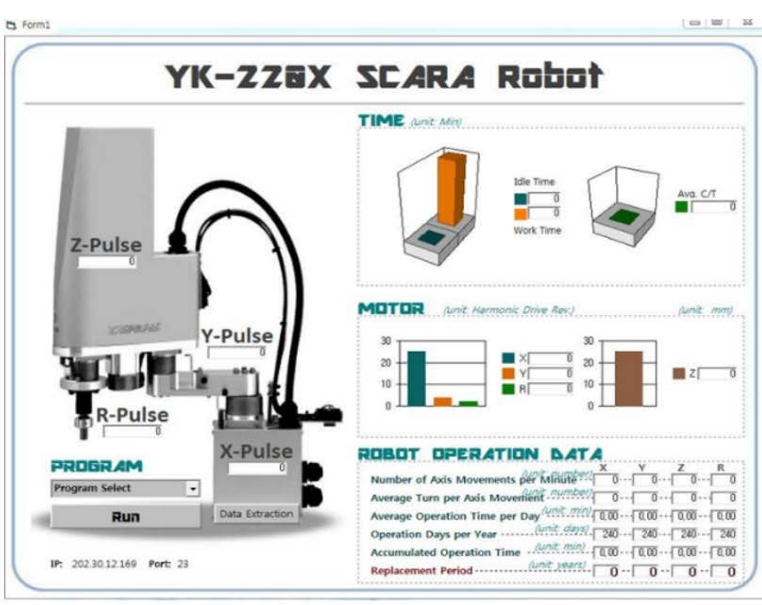

(b)

Figure 6. Snapshots of the developed API. (a) Integrated monitoring application; (b) Detailed robot status information.

Table 2. Comparative analysis for YK-220X robot: before improvement.

\begin{tabular}{ccccc}
\hline & \multicolumn{2}{c}{ Before Improvement-YK-220X } & & \\
\hline Axis & $\mathrm{X}$ & $\mathrm{Y}$ & $\mathrm{Z}$ & $\mathrm{R}$ \\
\hline No. of axis movements per min (n) & 10 & 21 & 15 & 9 \\
Average turn per axis movement $(\theta)$ & 0.06 & 0.18 & 0.07 & 0.10 \\
Speed (\%) & 100 & 100 & 100 & 100 \\
Speed reduction ratio (N) & 50 & 50 & 24 & 50 \\
Operation time/day (h) & 24 & 24 & 365 & 24 \\
Operation time/year (d) & 365 & $\mathbf{2}$ & $\mathbf{5}$ & 365 \\
Replacement period (years) & $\mathbf{9}$ & & & $\mathbf{6}$ \\
\hline
\end{tabular}




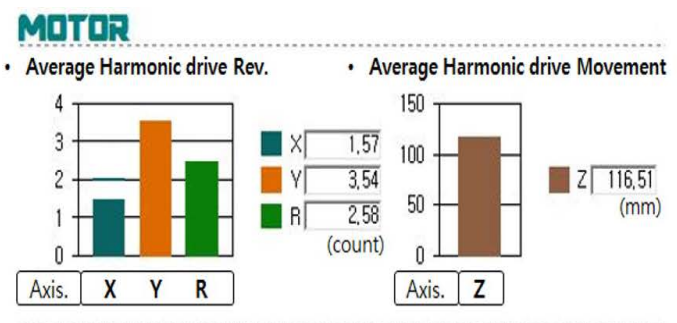

(a)

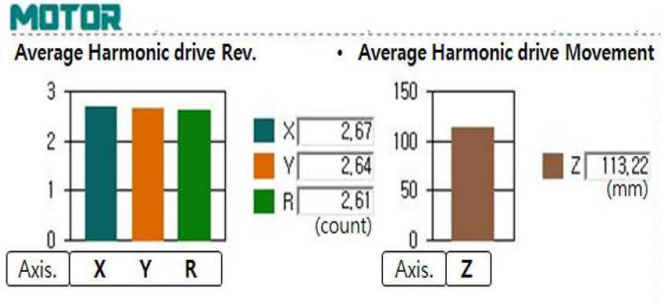

(b)

Figure 7. Actual usage of each axis for the YK-220X SCARA robot. (a) YK-220X robot: before improvement; (b) After improvement as a result of applying the quality informatics concept.

Table 3. Comparative analysis for YK-220X robot: after improvement.

\begin{tabular}{ccccc}
\hline & Before Improvement-YK-220X & & \\
\hline Axis & $\mathrm{X}$ & $\mathrm{Y}$ & $\mathrm{Z}$ & $\mathrm{R}$ \\
\hline No. of axis movements per min (n) & 11 & 18 & 15 & 5 \\
Average turn per axis movement $(\theta)$ & 0.07 & 0.05 & 0.06 & 0.15 \\
Speed (\%) & 100 & 100 & 100 & 100 \\
Speed reduction ratio (N) & 50 & 50 & $12 \mathrm{~mm}$ & 50 \\
Operation time/day (h) & 24 & 24 & 365 & 365 \\
Operation time/year (d) & 365 & 365 & $\mathbf{6}$ & $\mathbf{8}$ \\
Replacement period (years) & $\mathbf{7}$ & $\mathbf{6}$ & $\mathbf{6}$ & 24 \\
\hline
\end{tabular}

\section{Conclusion}

In the past, the proposed concept was difficult to implement due to technological limitations. With the advancement of ICT technologies, the production robots can be integrated within the information network, and the critical data can be processed to reveal the very important information. From the information, better managerial decisions are expected that will ultimately contribute to the organizations' efficiency and competitiveness. In this context, this study explains the merits of quality informatics in the domain of networked robots that are the essential tools for the global production companies. The future environment of global business will even push the boundaries of multinational, distributed production systems, and accelerate the implementation at a faster rate. Accordingly, the brand value of the product is expected to be greater than the country of the manufacturing. The survival of the company will be dependent on the optimized quality level that is encompassing the entire distributed production systems located throughout the whole. Under such circumstances, it can be foreseeable that the innovative ideas and the smart application of advanced technologies should be the priority.

\section{Acknowledgements}

This research was supported by the Basic Science Research Program through the National Research Foundation (NRF), funded by the Ministry of Education, Republic of Korea (Grant No. NRF-2013R1A1A2006108). The authors wish to express sincere gratitude for the financial support.

\section{References}

[1] Committee on Visionary Manufacturing (1998) Visionary Manufacturing Challenges for 2020. National Academic Press, Washington DC.

[2] Shi, J.J. and Zhou, S.Y. (2009) Quality Control and Improvement for Multistage Systems: A Survey. IIE Transactions, 41, 744-753. http://dx.doi.org/10.1080/07408170902966344

[3] Chan, H.K. (2011) Supply Chain Systems: Recent Trend in Research and Applications. IEEE Systems Journal, 5, 2-5. 
http://dx.doi.org/10.1109/JSYST.2010.2100191

[4] Stepanskiy, L. and Kwon, Y. (2010) Modeling of Robot Availability for the Network-Controllable Production System, Journal of Concurrent Engineering: Research \& Applications, 18, 303-310. http://dx.doi.org/10.1177/1063293X10389800

[5] Sarkisov, L. and Kim, J. (2013) Computational Structure Characterization Tools for the Era of Material Informatics. Special Issue on Molecular Modelling in Chemical Engineering, 121, 322-330. 